\title{
The Adult Respiratory Distress Syndrome Cognitive Outcomes Study: long-term neuropsychological function in survivors of acute lung injury
}

\author{
Catherine Gogela Carlson ${ }^{1,2}$ and David T Huang*1-3 \\ University of Pittsburgh Department of Critical Care Medicine: Evidence-Based Medicine Journal Club, edited by Sachin Yende
}

\begin{abstract}
Expanded abstract
Citation

Mikkelsen ME, Christie JD, Lanken PN, Biester RC, Thompson BT, Bellamy SL, Localio AR, Demissie E, Hopkins RO, Angus DC: The adult respiratory distress syndrome cognitive outcomes study: long-term neuropsychological function in survivors of acute lung injury. Am J Respir Crit Care Med 2012, 185:1307-1315.
\end{abstract}

\section{Background}

Cognitive and psychiatric morbidity is common and potentially modifiable after acute lung injury (ALI). However, practical measures of neuropsychological function for use in multicenter trials are lacking.

\section{Methods}

Objective: The objectives were to determine whether a validated telephone-based neuropsychological test battery is feasible in a multicenter trial and to determine the frequency and risk factors for long-term neuropsychological impairment.

Design: A prospective, multicenter cohort study of a subset of survivors from the Fluid and Catheter Treatment Trial (FACTT) was conducted.

Setting: The FACTT enrolled patients from 38 North American hospitals between June 2000 and October 2005. Subjects: To be eligible for the ALI Cognitive Outcomes Study (ACOS), subjects had to be enrolled in the FACTT and the EA-PAC (Economic Assessment of the Pulmonary Artery Catheter) trial. The FACTT enrolled mechanically ventilated adults who met the AmericanEuropean Consensus Conference criteria for ALI.

*Correspondence: huangdt@upmc.edu

'Department of Critical Care Medicine, University of Pittsburgh, 3550 Terrace Street, 6th floor, Pittsburgh, PA 15261, USA

Full list of author information is available at the end of the article
Intervention: In an adjunct study to the Acute Respiratory Distress Syndrome Clinical Trials Network Fluid and Catheter Treatment Trial, neuropsychological function at 2 and 12 months after hospital discharge was assessed.

Outcomes: The primary outcome was the result of a validated telephone battery of standardized neuropsychological tests administered to consenting, English-speaking subjects at 2 and 12 months after hospital discharge.

\section{Results}

Of 406 eligible survivors, 261 patients were approached to participate and 213 consented. One hundred twenty-two subjects, including 102 subjects at 12 months, were tested at least once. Memory, verbal fluency, and executive function were impaired in 13\% (12 of 92), 16\% (15 of 96), and 49\% (37 of 76) of long-term survivors, respectively. Long-term cognitive impairment was present in 41 (55\%) of the 75 survivors who completed cognitive testing. Depression, post-traumatic stress disorder, and anxiety were present in 36\% (37 of 102), 39\% (40 of 102), and 62\% (63 of 102) of long-term survivors, respectively. Enrollment in a conservative fluid management strategy $(P<0.005)$ was associated with cognitive impairment, and lower partial pressure of arterial oxygen during the trial was associated with cognitive $(P<0.02)$ and psychiatric $(P<0.02)$ impairment.

\section{Conclusions}

Neuropsychological function can be assessed by telephone in a multicenter trial. Long-term neuropsychological impairment is common in survivors of ALI. Hypoxemia is a risk factor for long-term neuropsychological impairment. A fluid management strategy is a potential risk factor for long-term cognitive impairment; however, given the select population studied and an unclear mechanism, this finding requires confirmation.

\section{Commentary}

Acute lung injury (ALI), now referred to as 'mild acute respiratory distress syndrome (ARDS)' [1], is common in 
the critical care setting. Neuropsychiatric morbidities are prevalent and long-lasting in survivors of critical illness [2]. With a growing number of ALI survivors, more effort is being directed toward understanding and improving the long-term physical, cognitive, and psychiatric morbidities experienced by these survivors. The Fluid and Catheter Treatment Trial (FACTT) suggested shortterm physiologic benefit from a conservative fluid management strategy when compared with a liberal fluid management strategy, as evidenced by improved lung function and shorter duration of mechanical ventilation $[3,4]$. In the FACTT, the conservative strategy group had better lung injury scores and oxygenation indices as well as lower plateau pressures and positive end-expiratory pressures. However, there was no difference in hospital mortality at 60 days between the two treatment groups.

As a concurrent study to the FACTT, the ALI Cognitive Outcomes Study (ACOS) demonstrated a high incidence of cognitive impairment and psychiatric symptoms in long-term survivors of ALI. Fifty-five percent of patients had cognitive impairment on neuropsychological testing. However, cognitive impairment was significantly more common in the fluid conservative arm $(P=0.005)$, particularly in executive dysfunction domains (such as planning a trip or balancing a checkbook). In the ACOS, enrollment in the conservative fluid management strategy $(P=0.004)$ and lower partial pressure of arterial oxygen $(P=0.02)$ were associated with cognitive impairment at 12 months, suggesting that the short-term benefits achieved as a result of a conservative fluid strategy might come at the cost of long-term cognitive dysfunction. The results of this study illustrate that physiologic outcomes are not necessarily associated with patient-centered outcomes.

The ACOS is the first study to determine whether neuropsychological function can be assessed in a multicenter trial. It is also the first large-scale assessment of a previously validated telephone-administered neuropsychological instrument. However, one weakness of the study is its small sample size, a consequence of high patient attrition and low telephone enrollment. Several possible explanations account for this lack of follow-up. For instance, the subject may have been lost to follow-up because of death, known as a competing risk. It is also possible that the subject was lost to follow-up because of neurocognitive deficits, known as informative censoring. Although bias from pre-study cognitive baseline should have been addressed by randomization, unmeasured differences could have resulted in differential attrition between study arms. Non-informative censoring, or random loss to follow-up, is another potential explanation for the high patient attrition in this study.

\section{Recommendation}

Consistent with previous studies, the ACOS showed neurocognitive deficits in a large proportion of subjects recovering from ARDS (55\% of patients had cognitive impairment on neuropsychiatric testing). Future studies should incorporate more long-term follow-up, as benefits of intensive care unit interventions observed during the early part of recovery from critical illness may not translate into improvement in long-term outcomes.

\section{Abbreviations}

ACOS, Acute Lung Injury Cognitive Outcomes Study; ALI, acute lung injury; ARDS, acute respiratory distress syndrome; FACTT, Fluid and Catheter Treatment Trial.

\section{Competing interests}

The authors declare that they have no competing interests.

\section{Author details}

'Department of Critical Care Medicine, University of Pittsburgh, 3550 Terrace Street, 6th floor, Pittsburgh, PA 15261, USA. ${ }^{2}$ Department of Emergency Medicine, University of Pittsburgh, Suite 10028 Forbes Tower, Pittsburgh, PA 15260, USA. ${ }^{3}$ The Clinical Research, Investigation, and Systems Modeling of Acute Illness (CRISMA) Center, University of Pittsburgh, 3550 Terrace Street, 6th floor, Pittsburgh, PA 15261, USA.

Published: 24 May 2013

\section{References}

1. ARDS Definition Task Force, Ranieri VM, Rubenfeld GD, Thompson BT, Ferguson ND, Caldwell E, Fan E, Camporota L, Slutsky AS: Acute respiratory distress syndrome: the Berlin definition. JAMA 2012, 307:2526-2533.

2. Hopkins RO, Weaver LK, Pope D, Orme JF, Bigler ED, Larson-Lohr V: Neuropsychological sequelae and impaired health status in survivors of severe acute respiratory distress syndrome. Am J Respir Crit Care Med 1999, 160:50-56.

3. National Heart, Lung, and Blood Institute Acute Respiratory Distress Syndrome (ARDS) Clinical Trials Network, Wiedemann HP, Wheeler AP, Bernard GR, Thompson BT, Hayden D, deBoisblanc B, Connors AF Jr., Hite RD, Harabin AL: Comparison of two fluid management strategies in acute lung injury. N Engl J Med 2006, 354:2564-2575.

4. National Heart, Lung, and Blood Institute Acute Respiratory Distress Syndrome (ARDS) Clinical Trials Network, Wheeler AP, Bernard GR, Thompson BT, Schoenfeld D, Wiedemann HP, deBoisblanc B, Connors AF Jr., Hite RD, Harabin AL: Pulmonary-artery versus central venous catheter to guide treatment of acute lung injury. N Engl J Med 2006, 354:2213-2224.

\section{doi:10.1186/cc12709}

Cite this article as: Gogela Carlson C, Hunag DT: The Adult

Respiratory Distress Syndrome Cognitive Outcomes Study: long-term neuropsychological function in survivors of acute lung injury. Critical Care 2013, 17:317. 